\title{
Large renal leiomyoma: A multidisciplinary approach to diagnosis
}

\author{
Sotiris Chaniotakis ${ }^{1 *}$, Yi Yang ${ }^{2}$, Tulsi Patel ${ }^{2}$, James Banks ${ }^{1,2}$ \\ 1. Nova Southeastern University College of Medicine, Florida, USA \\ 2. Aventura Hospital and Medical Center, Florida, USA \\ * Correspondence: Sotiris Chaniotakis M.S., Nova Southeastern University, 562 NW 97th Ave, Plantation, FL, 33324, USA \\ (\schaniot@gmail.com) \\ Radiology Case. 2021 Aug; 15(8):18-26 $\quad$ :: $\quad$ DOI: 10.3941/jrcr.v15i8.4125
}

\begin{abstract}
We report the case of a 45-year-old woman who presented with a large palpable abdominal mass. Initial sonographic and computed tomographic studies prompted a differential diagnosis of retroperitoneal or renal sarcoma, leiomyoma, and lipid-poor angiomyolipoma. A final diagnosis of renal leiomyoma was reached based on a consensus among radiology, surgery and pathology. In addition to reviewing the features of this entity, this case demonstrates the process of developing a working diagnosis, narrowing the differential as additional testing is performed and establishing a final diagnosis with interdepartmental coordination. Despite the rarity of this condition, the ability to recognize and apply imaging features to differentiate between abdominal masses of unknown origin is important for clinicians and researchers.
\end{abstract}

\section{CASE REPORT}

\section{CASE REPORT}

A 45-year-old female with a vague past medical history of an indeterminate liver mass came to the emergency department complaining of 2 weeks of sharp intermittent right upper quadrant abdominal pain occasionally radiating to her right flank that suddenly worsened in severity to 8/10 that day. Her pain was not associated with eating. She denied fevers, chills, jaundice, gastrointestinal, genitourinary, or cardiovascular complaints. On physical exam, her vital signs were normal, and a large, mobile, tender mass was palpated in the right upper quadrant of the abdomen. Abdominal ultrasound in the emergency department (Fig. 1) identified a solid, circumscribed mass in the right hemiabdomen that extended beyond the full field of view.
A subsequent axial computed tomography (CT) scan with and without intravenous (IV) contrast was performed (Fig. 2) which demonstrated a heterogeneous, noncalcified right retroperitoneal mass without fat, arising from the upper pole of the right kidney. In the non-contrast $\mathrm{CT}$ of the abdomen with axial reformats in liver window (Fig. 2A), multiple regions of interest were sampled with the average Hounsfield unit ranging from 33 to 48 (SD of 16-18) with no evidence of macroscopic fat. The mass was well-circumscribed and appeared to be separated from the liver by a fat plane.

Contrast enhanced CT of the abdomen in the portal phase with axial reformats in soft tissue window (Fig. 2B) demonstrated the mass to be heterogeneously enhancing with a few foci of avid enhancement suggesting vascularity. The mass was hypoenhancing relative to the right renal cortex and appeared inseparable from the right kidney with a "claw sign" 
splaying the renal sinus with the epicenter of the tumor therefore likely in the right kidney upper pole. The "claw sign" suggested a neoplasm of renal, urothelial or renovascular origin. There was significant mass effect on the residual right kidney, the other retroperitoneal structures, the liver and inferior vena cava. There was no evidence of metastasis, hydronephrosis, or invasion of the chest wall, diaphragm, and adjacent organs.

Contrast enhanced coronal reconstruction in soft tissue window demonstrated heterogeneous enhancement (Fig. 3A) with several foci of avid enhancement, more conspicuous on coronal reconstructed maximum intensity projection images (Fig. 3B). These foci appeared to represent intra-tumoral vasculature. The mass replaced the right kidney upper pole again displaying the "claw sign" (Fig. 3A), and appeared to emerge from the cortex or capsule of the upper pole of the right kidney and extended rostrally. The liver appeared separate from the mass with inferior vena cava displacement to the left (Fig 3A). The superior border of the mass was delineated by the right hemidiaphragm without evidence of invasion (Fig 3A). The lateral border of the mass abutted ribs 9-12 without evidence of chest wall invasion. The medial border of the mass abutted the T9 to L1 vertebral bodies and the right psoas muscle without evidence of invasion. Several enlarged vessels were identified in the retroperitoneum inferior to the mass, presumed to be engorged collateral veins secondary to mass effect on the vena cava (Fig 3B).

Aortogram and renal angiogram (Fig. 4A-C) demonstrated the arterial supply of the tumor arose from right renal artery superior and anterior branches. There was an absence of normal right upper pole renal cortical enhancement compatible with tumor replacing this portion of the kidney. Numerous tortuous and abnormal vessels were identified throughout the mass confirming the presence of vascularity seen on CT. The central area of the tumor appeared to be poorly perfused by this study and may indicate that this area is poorly vascularized.

Abdominal aortogram (Fig. 4A) demonstrated normal perfusion to the left kidney and no abnormalities of the aorta. Renal angiogram (Fig. 4B-C) displayed multiple tortuous and irregular vessels within the tumor but without aneurysm. No cortical parenchymal perfusion was identified within the superior pole of the kidney and there was no evidence of venous drainage or excretion on the delayed images (Fig. 4C).

The patient underwent diagnostic laparoscopy to inspect tumor margins, exclude peritoneal metastasis, and to confirm radiologic findings that favored a benign tumor. The intraoperative report described an easily ballotable soft tissue tumor arising from the right retroperitoneum. The mass was described as separable from but exerting mass effect on the liver. There were extensive varices surrounding the tumor. No signs of peritoneal disease or visceral surface metastatic deposits were identified by gross inspection of the viscera during surgery. Fluid collected from the peritoneal cavity and subdiaphragmatic space was sent for cytology and revealed no malignant cells. Due to the location of the tumor and high risk of variceal bleeding, intraoperative biopsy was not conducted.
Instead, an ultrasound guided percutaneous core needle biopsy was performed with histologic sections of the pink-tan tissue demonstrating a well-differentiated smooth muscle neoplasm (Fig. 5) with no evidence of malignancy. The cells stained positive for Desmin and smooth muscle actin (SMA) (Fig. 6, Fig. 7) and negative for S100, Ckit, Pankeratin and HMB-45. The Ki-67 staining demonstrated a very low proliferation rate (Fig. 8). A diagnosis of renal leiomyoma was reached and the patient was advised to have the tumor removed. She elected to have the procedure elsewhere and was lost to follow-up.

\section{DISCUSSION}

\section{Etiology \& Demographics:}

Small, clinically insignificant renal leiomyomas are suspected to be relatively common and have been reported to be found in 4.2 to 5.2 percent of autopsies [1]. Despite this, they account for only $0.29 \%$ of all treated renal tumors as the vast majority are asymptomatic and are discovered incidentally. Clinically significant large renal leiomyomas such as the one identified in this case are exceptionally rare. Renal leiomyomas are regular, well-circumscribed, encapsulated benign lesions of well-differentiated smooth muscle cells [2]. On gross examination they are firm, whirling, white or red masses. It has been shown that they typically arise from smooth muscle cells within the renal capsule or renal pelvis or sometimes from the tunica media of renal vasculature [3]. Renal leiomyomas display a female predilection in a ratio of $2: 1$, which may be partially explained by the fact that these tumors have been shown to uniformly express estrogen and progesterone receptors [3,4]. In support of this, renal leiomyomas occur most commonly in women of reproductive age, at which time hormonal profiles may foster supportive conditions for tumor growth [5]. The mean age of presentation is 42 years [1]. Similar to the presentation in this case, these benign lesions are typically asymptomatic until they exhibit mass effect. This is consistent with the large average reported size of leiomyomas in the retroperitoneum of $16.2 \mathrm{~cm}$ in length and $1.6 \mathrm{~kg}$ in weight [6]. The mass in our patient measured at least $20.6 \mathrm{~cm}$ by imaging, comparable but larger than the reported average. Studies on leiomyomas in other locations of the body have shown that metastasis is a very rare occurrence [7].

\section{Clinical \& Imaging findings:}

Renal leiomyomas typically appear as hypoechoic solid masses on ultrasound. In non-contrast CT, these tumors are typically hyperdense compared to the renal parenchyma and exhibit comparable density to muscle. In CT with contrast, leiomyomas are hypoenhancing compared to the renal parenchyma at the corticomedullary phase and show progressive enhancement in later phases. Most enhance homogeneously, however, larger masses, as in our case, can enhance heterogeneously possibly due to degeneration and necrosis. It has been reported that renal leiomyomas display low signal intensity on magnetic resonance imaging (MRI) for both T1- and T2-weighted images [8]. Most renal leiomyomas are asymptomatic, however, when large enough, they often present with flank and/or abdominal pain and a palpable mass, while less frequently with hematuria [1]. 


\section{Treatment \& Prognosis:}

Surgical resection is the gold standard treatment for large, clinically significant renal leiomyomas; sometimes excision is possible with renal sparing operations $[9,10]$. Small, indolent masses of the kidney discovered incidentally often do not require treatment, and watchful waiting may be appropriate. The prognosis after surgery is excellent with low risk of recurrence. A study by Gupta et al. (2016) showed that of 10 patients who were followed up after the surgical excision of a renal leiomyoma, none had a tumor recurrence [4].

\section{Differential Diagnoses:}

The physical exam demonstrated a large mobile, palpable mass in the right upper quadrant. Masses palpated under the right ribs could arise from the liver, gallbladder, bowel, right kidney, adrenal gland, vasculature or intra-abdominal fat. Retroperitoneal tumors must be very large to be detected on physical exam and would therefore be considered less likely. This line of reasoning likely led to confusion initially as the patient presented with a reported history of a "liver mass".

Ultrasound findings show a large solid mass, with varying echogenicity (Fig. 1). Deep seated masses of this size are notoriously difficult to fully characterize by ultrasound, with the location of a mass and its relationship to adjacent structures often requiring CT or MRI to accurately depict. The presence of heterogeneous echotexture, vascularity and posterior acoustic shadowing raised suspicion for malignancy although the borders of the mass appeared circumscribed. Based on the ultrasound alone, the possibility of a liver lesion as reported by the patient, although unlikely given the imaging appearance, was not refuted.

According to the American College of Radiology (ACR) Appropriateness Criteria, abdominal ultrasound and abdominal CT with IV contrast are the most appropriate initial studies for a palpable abdominal mass suspected to be a neoplasm [10]. In accordance with this, subsequent CT studies confidently placed the mass within the retroperitoneum and separable from the liver (Fig. 2, Fig. 3). This was confirmed during diagnostic laparoscopy noting that the liver and the mass were easily separable with an intact dorsal portion of the parietal peritoneum. Despite these studies, reference to a "liver mass" in the emergency department note persisted in subsequent clinical notes and diagnosis related group codes likely a result of anchoring bias. Anchoring bias is a form of cognitive bias in which information that was learned at an early stage is overvalued and affects future decision making that has been shown to be prevalent even among experts within a field [11].

Loss of the normal right kidney upper pole replaced by the mass as seen on CT and angiography (Fig 3.A, Fig. 4), can be caused by an extra-renal mass with local invasion or a tumor arising from the kidney. The claw sign is useful for distinguishing these possibilities. The claw sign refers to the acute margins of renal parenchyma with an indistinct outline situated on either side of a portion of the tumor, indicating expansile growth of a mass from that organ (Fig. 2B, Fig 3A). A mass arising from a separate structure would produce obtuse margins with the adjacent parenchyma deformed by extrinsic compression. Although the heterogeneity of the mass could be concerning for a sarcoma, the presence of a defined capsule and absence of local or regional metastasis favored a benign etiology such as leiomyoma or angiomyolipoma (AML). Based on the imaging findings alone, however, a retroperitoneal or renal sarcoma, carcinoma, or solitary fibrous tumor could not be excluded. The possibility that the mass was of adrenal origin was excluded based on the angiographic findings.

Diagnostic laparoscopy confirmed the absence of locoregional invasion and the absence of malignant cells in peritoneal washings. Biopsy confirmed the tumor of smooth muscle origin (Fig. 5, Fig. 6, Fig. 7) which prompted the narrowing of the broad diagnosis of sarcoma to leiomyosarcoma.

\section{Leiomyosarcoma}

Renal leiomyosarcomas are highly malignant neoplasms of smooth muscle cells accounting for less than $3 \%$ of renal malignancies. They present late with abdominal/lumbar pain, palpable mass, vomiting, hematuria and weight loss [12] and are usually fatal (29\%-36\% 5 year survival rate) [13]. They enhance heterogeneously on CT, commonly with evidence of hemorrhage, necrosis and cystic change. With MRI, leiomyosarcomas have heterogeneous signal intensity with areas of low signal intensity on T1- and T2-weighted images. On ultrasound they display heterogeneous echotexture - a more detailed consensus on the specific ultrasound characteristics of renal leiomyosarcomas has not been reached [14].

\section{Lipid-Poor Angiomyolipoma}

The hyperattenuating nature of the mass relative to the kidney but not containing macroscopic fat (Fig. 2A) essentially excluded lipid rich AML, but did not rule out lipidpoor AML. AMLs are common benign tumors composed of some combination of smooth muscle, vascular, and adipose tissue. AMLs are highly associated with tuberous sclerosis complex (TSC), an autosomal dominant genetic disorder that causes renal AMLs in approximately $80 \%$ of patients with this condition [15]. Similar to leiomyomas, asymptomatic AMLs are common, with a prevalence of $0.44 \%$ in healthy individuals [16]. They are often found incidentally, however they can present with flank pain, hematuria, spontaneous retroperitoneal hemorrhage, anemia, urinary tract infection and renal failure [17]. While the classification of AMLs is an ongoing debate, the most widely used sub-categorization scheme is based on the amount of fat detectable with radiography. Fat-poor AML is differentiated from classic AML by having no evidence of macroscopic fat on unenhanced CT. Fat-poor AMLs are further classified into hyperattenuating subtype, isoattenuating subtype and AML with epithelial cysts. The CT findings in the present case favor a diagnosis of fat-poor AML hyperattenuating subtype. These tumors represent $4-5 \%$ of all sporadic AMLs. Fat-poor AML hyperattenuating subtype mimics the imaging findings of leiomyoma in many ways as both are benign and composed primarily of well differentiated smooth muscle. They are hyperattenuating relative to the renal parenchyma on unenhanced CT, T1 and T2 hypointense, and appear isoechoic on ultrasound [18]. At pathology they contain very little fat $(\sim 4 \%)$ and mostly smooth muscle [19]. 
Percutaneous core biopsy of the retroperitoneal mass demonstrated well-differentiated smooth muscle cells without atypia, a low mitotic index, and no evidence of necrosis (Fig. 5). Sparse Ki-67 antibody staining (Fig. 8) demonstrated very low cellular proliferation confirming that the mass was not a leiomyosarcoma. Immunohistochemistry was then useful for differentiating between leiomyoma and fat-poor AML hyperattenuating subtype. The cells stained positive for Desmin and SMA, and negative for S100, Ckit, Pankeratin and HMB-45. Research suggests this profile supports the diagnosis of leiomyoma over AML. In one study, all biopsy samples from patients with AMLs were found to be positive for HMB-45 while HMB-45 immunoreactivity was negative for all leiomyomas, with a significant $(\mathrm{P}<0.001)$ association between AMLs and HMB-45 immunoreactivity [20]. Another study found positive HMB-45 immunoreactivity in 17 out of 19 cases of renal AMLs [21]. A third study analyzing 15 cases of renal AML found HMB-45 reactivity in all cases [22]. HMB-45 has been proposed as the best immunohistochemical marker for confirming the diagnosis of AML by yet another study [23]. Negative immunoreactivity for HMB-45 in our case suggests against the diagnosis of AML. In addition, C-kit immunoreactivity which has been shown to be associated with AML, was also negative in our case [24]. Positive SMA, Desmin and negative S100 staining profiles have significant overlap between AML and leiomyoma, and therefore are not useful for differentiating between these two differential diagnoses [25].

It is important to note that the core biopsy taken represents only a very small proportion of a massive and potentially heterogeneous tumor. It is possible that the area sampled contained different cellular characteristics not representative of the tumor as a whole. Despite this caveat, histopathology virtually confirms a diagnosis of renal leiomyoma which is concordant with the imaging and laparoscopic assessment.

\section{Conclusion:}

A differential diagnosis of renal leiomyoma, fat-poor AML hyperattenuating subtype, and renal leiomyosarcoma was developed. Radiological, surgical, and pathological coordination was crucial for the accurate diagnosis of renal leiomyoma. While strong evidence can be raised to support a diagnosis by synthesizing information from the history and physical, imaging and surgical report, pathology is required for diagnostic confirmation. The diagnostic approach presented can be added to the resources clinicians and researchers have at their disposal for differentiating between these rare conditions.

\section{TEACHING POINT}

Renal Leiomyomas are usually small benign neoplasms of well differentiated smooth muscle that rarely cause symptoms. When large and symptomatic, they appear regular, distinct and encapsulated in appearance on ultrasound and CT, hypoenhance with IV contrast relative to the renal parenchyma and display no evidence of macroscopic fat.

\section{REFERENCES}

1. Khetrapal S, Bhargava A, Jetley S, Rana S, Jairajpuri Z. Renal leiomyoma: an uncommon differential diagnosis of renal masses with a clinical relevance. J Clin Diagn Res. 2014 Oct;8(10):FD08-9. PMID: 25478354

2. Mak CW, Chou CK, Tzeng WS. Renal capsular leiomyoma: Report of a case with unusual CT appearance. Acta Radiol. 2006;47(7):752?754. PMID: 16950717

3. Larbcharoensub N, Limprasert V, Pangpunyakulchai D, Sanpaphant S, Wiratkapun C, Kijvikai K. Renal Leiomyoma: A Case Report and Review of the Literature. Urology Case Reports. 2017;13:3-5. PMID: 28417074

4. Gupta S, Jimenez RE, Folpe AL, Cheville JC. Renal Leiomyoma and Leiomyosarcoma: A Study of 57 Cases. Am J Surg Pathol. 2016;40(11):1557?1563. PMID: 27276240

5. Fasih N, Prasad Shanbhogue AK, Macdonald DB, et al. Leiomyomas beyond the Uterus: Unusual Locations, Rare Manifestations. RadioGraphics. 2008;28(7):1931-1948. PMID: 19001649

6. Paal E, Miettinen M. Retroperitoneal leiomyomas: a clinicopathologic and immunohistochemical study of 56 cases with a comparison to retroperitoneal leiomyosarcomas. Am J Surg Pathol. 2001;25(11):1355?1363. PMID: 11684951

7. Barnaś E, Książek M, Raś R, Skręt A, Skręt- Magierło J, Dmoch- Gajzlerska E. Benign metastasizing leiomyoma: A review of current literature in respect to the time and type of previous gynecological surgery. Robboy SJ, ed. PLOS ONE. 2017;12(4):e0175875. PMID: 28426767

8. Onur MR, Akin MM, Onur AR. Renal Leiomyoma: Ultrasonography and Computed Tomography Features with Histopathologic Correlation. The Eurasian Journal of Medicine. 2013;45(3):214-217. PMID: 25610282

9. Brunocilla E, Pultrone CV, Schiavina R, Vagnoni V, Caprara G, Martorana G. Renal leiomyoma: Case report and literature review. Canadian Urological Association Journal. 2012;6(2):E87-E90. PMID: 22511443

10. Fowler KJ, Garcia EM, Kim DH, et al. ACR Appropriateness Criteria ${ }^{\circledR}$ Palpable Abdominal MassSuspected Neoplasm. Journal of the American College of Radiology. 2019;16(11):S384-S391. PMID: 31685106

11. Saposnik G, Redelmeier D, Ruff CC, Tobler PN. Cognitive biases associated with medical decisions: a systematic review. BMC Med Inform Decis Mak. 2016 Nov 3;16(1):138. PMID: 27809908

12. Beardo P, José Ledo M, Jose Luis RC. Renal Leiomyosarcoma. Rare Tumors. 2013;5(3):144-145. PMID: 24179654

13. Katabathina VS, Vikram R, Nagar AM, Tamboli P, Menias CO, Prasad SR. Mesenchymal neoplasms of the kidney in adults: imaging spectrum with radiologic-pathologic 
correlation. Radiographics. 2010 Oct;30(6):1525-40. PMID: 21071373

14. Venkatesh K, Lamba Saini M, Niveditha SR, Krishnagiri C, Babu S. Primary Leiomyosarcoma of the Kidney. Pathology Research International. 2010;2010:1-4. PMID: 21151722

15. Ni J, Yan F, Qin W, et al. Mutational analysis of renal angiomyolipoma associated with tuberous sclerosis complex and the outcome of short-term everolimus therapy. Scientific Reports. 2019;9(1). PMID: 31586081

16. Fittschen A, Wendlik I, Oeztuerk S, et al. Prevalence of sporadic renal angiomyolipoma: a retrospective analysis of 61,389 in- and out-patients. Abdominal Imaging. 2014;39(5):1009-1013. PMID: 24705668

17. Unlu C. Retroperitoneal haemorrhage caused by a renal angiomyolipoma. Emergency Medicine Journal. 2006;23(6):464-465. PMID: 16714510

18. Jinzaki M, Silverman SG, Akita H, Nagashima Y, Mikami S, Oya M. Renal angiomyolipoma: a radiological classification and update on recent developments in diagnosis and management. Abdominal Imaging. 2014;39(3):588-604. PMID: 24504542

19. Jinzaki M, Silverman SG, Akita H, Mikami S, Oya M. Diagnosis of Renal Angiomyolipomas: Classic, Fat-Poor, and Epithelioid Types. Seminars in Ultrasound, CT and MRI. 2017;38(1):37-46. PMID: 28237279

20. Yaldiz M, Kilinc N, Ozdemir E. Strong association of HMB-45 expression with renal angiomyolipoma. Saudi Med J. 2004;25(8):1020?1023. PMID: 15322591

21. Ashfaq R, Weinberg AG, Albores-Saavedra J. Renal angiomyolipomas and HMB-45 reactivity. Cancer. 1993;71(10):3091?3097. PMID: 8490837

22. Esheba GES, Esheba NES. Angiomyolipoma of the kidney: Clinicopathological and immunohistochemical study. Journal of the Egyptian National Cancer Institute. 2013;25(3):125-134. PMID: 23932749

23. Makhlouf HR, Ishak KG, Shekar R, Sesterhenn IA, Young DY, Fanburg-Smith JC. Melanoma markers in angiomyolipoma of the liver and kidney: a comparative study. Arch Pathol Lab Med. 2002;126(1):49?55. PMID: 11800647

24. Makhlouf HR, Remotti HE, Ishak KG. Expression of KIT (CD117) in angiomyolipoma. Am J Surg Pathol. 2002;26(4):493?497. PMID: 11914628

25. McCarthy AJ, Chetty R. Benign Smooth Muscle Tumors (Leiomyomas) of Deep Somatic Soft Tissue. Sarcoma. 2018 Sep 9;2018:2071394. PMID: 30271265

\section{FIGURES}

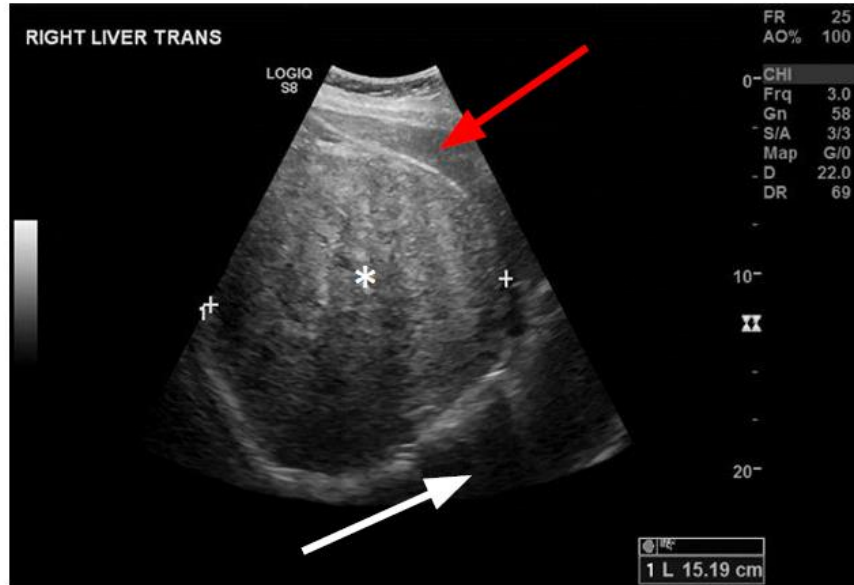

Figure 1: 45 year-old woman with renal leiomyoma.

FINDINGS: Well circumscribed encapsulated mass with borders denoted by measurement calipers, extending beyond the full field of view. Heterogeneous echotexture (asterisk) including areas of linear shadowing within the mass give a "venetian blind appearance". Posterior shadowing (white arrow) confirms solidity. Liver is displaced medially (red arrow).

TECHNIQUE: Abdominal Ultrasound in transverse plane using $\mathrm{C} 1-5$ convex probe at $3 \mathrm{MHz}$. 

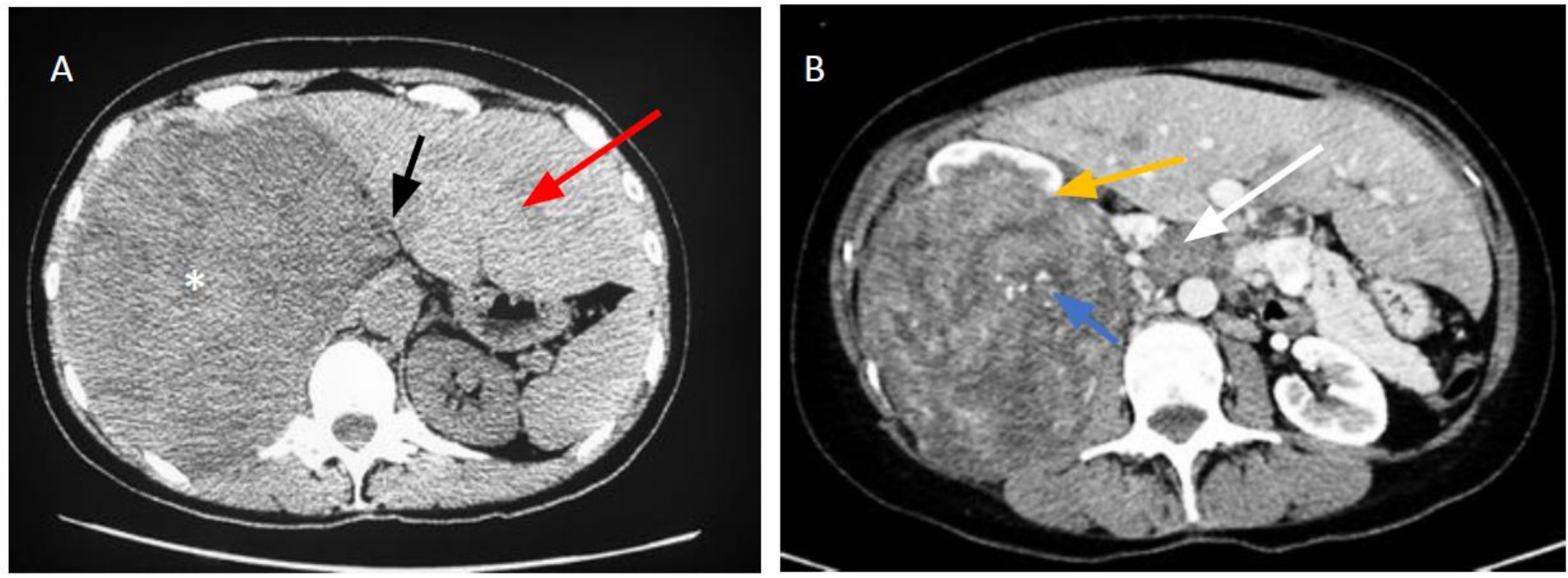

Figure 2: 45 year-old woman with renal leiomyoma.

FINDINGS: 2A) Non contrast CT demonstrating retroperitoneal tumor (asterisk) with no evidence of macroscopic fat or calcification. Red arrow indicates the liver which is displaced by mass effect into the left hemiabdomen. Black arrow indicates fat plane separating the mass from the liver.

2B) Yellow arrow shows claw sign indicating expansile growth of the mass from the kidney. Blue arrow demonstrates numerous intratumoral arterial enhancing vessels. White arrow indicates left displaced vena cava.

TECHNIQUE: 2A) Non-contrast CT of abdomen with axial reformats in liver window (439 mAs; Exp time 600; 120 kVP; slice thickness $2.5 \mathrm{~cm}$; pitch 1.375:1; slice space 3.4375 ).

2B) Contrast enhanced CT in late arterial phase with axial reformats in soft tissue window (170 mA; Exp time 700 ; $100 \mathrm{kVP}$; slice thickness $2.5 \mathrm{~cm}$; pitch 1.375:1; slice space 4.125; 100mL Isovue $3704 \mathrm{~mL} / 40 \mathrm{sec}$ ).
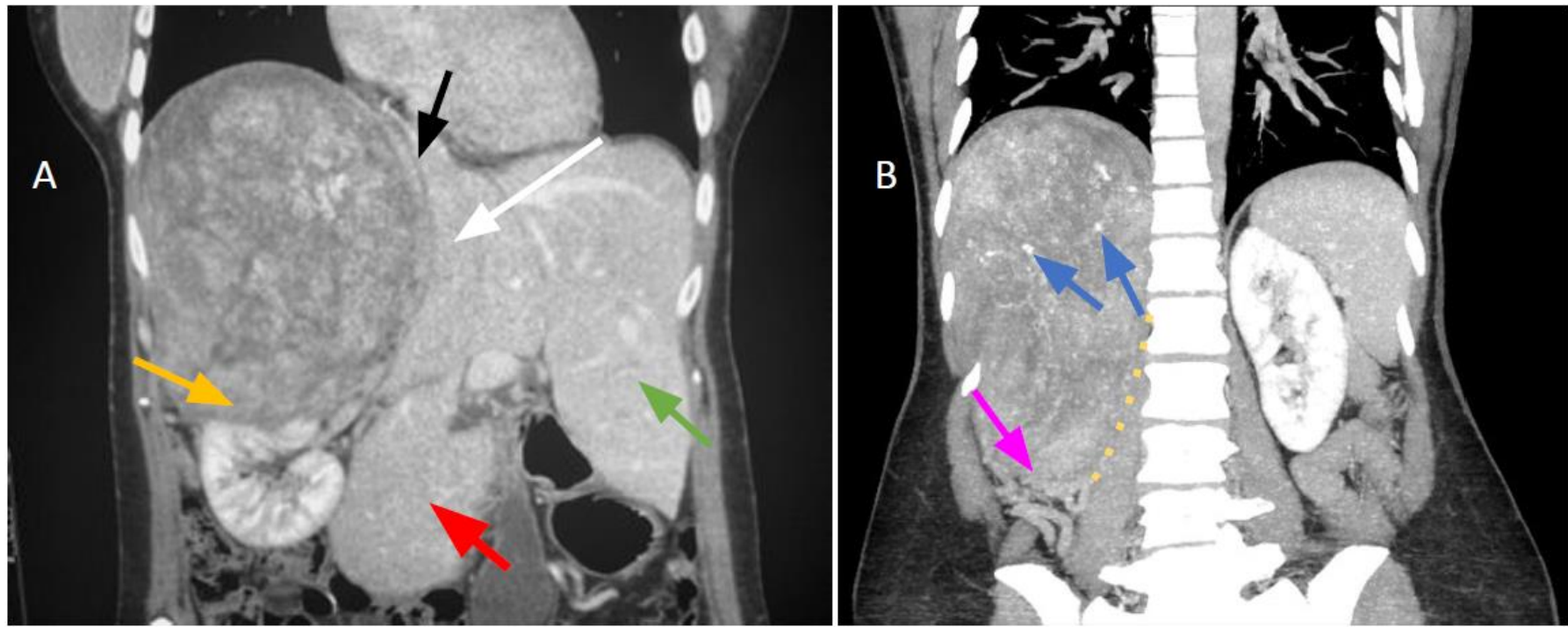

Figure 3: 45 year-old woman with renal leiomyoma.

FINDINGS: 2A) Orange arrow indicates claw sign. Red arrow indicates the right lobe of the liver. White arrow indicates a compressed but patent IVC. Black arrow indicates a fat plane between the mass and the IVC. The mass measures $20.6 \mathrm{~cm}$ in length.

2B) Blue arrows indicate blood filled spaces with delayed washout. Dashed yellow line roughly delineates the medial aspect of the mass and separates it from the right psoas muscle. Pink arrow demonstrates engorged veins.

TECHNIQUE: 2A) Contrast enhanced CT in the delayed phase with coronal reconstruction in soft tissue window (170 mA; Exp time 700; $100 \mathrm{kVP}$; slice thickness 2.5cm; pitch 1.375:1; slice space 4.125; $100 \mathrm{~mL}$ Isovue $3704 \mathrm{~mL} / 40 \mathrm{sec}$ ).

2B) Contrast enhanced CT in the delayed phase with coronal maximum intensity projection reconstruction in soft tissue window (170 mA; Exp time 700; $100 \mathrm{kVP}$; slice thickness 2.5cm; pitch 1.375:1; slice space 4.125; 100mL Isovue $3704 \mathrm{~mL} / 40 \mathrm{sec}$ ). 

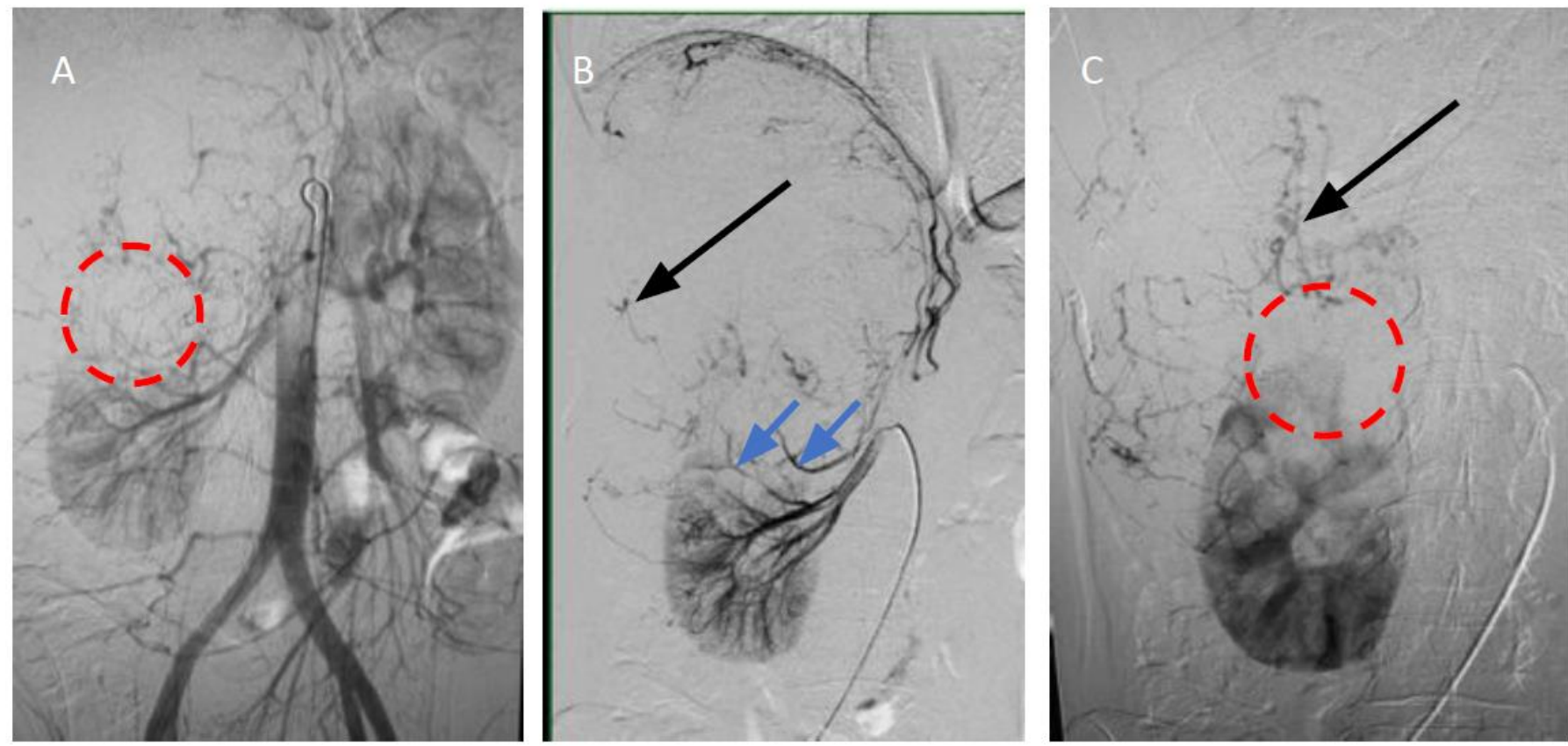

Figure 4: 45 year-old woman with renal leiomyoma.

FINDINGS: Red dashed circle displays absence of normal right upper pole renal cortical enhancement on early and delayed phases. Blue arrows point to the anterior and superior branches of the right renal artery supplying the tumor. Black arrow indicates tortuous and abnormal vessels within the mass.

TECHNIQUE: Abdominal aortogram (A), Renal arteriogram (B) and Renal nephrogram (C) (19 mAs, 80 kVP)

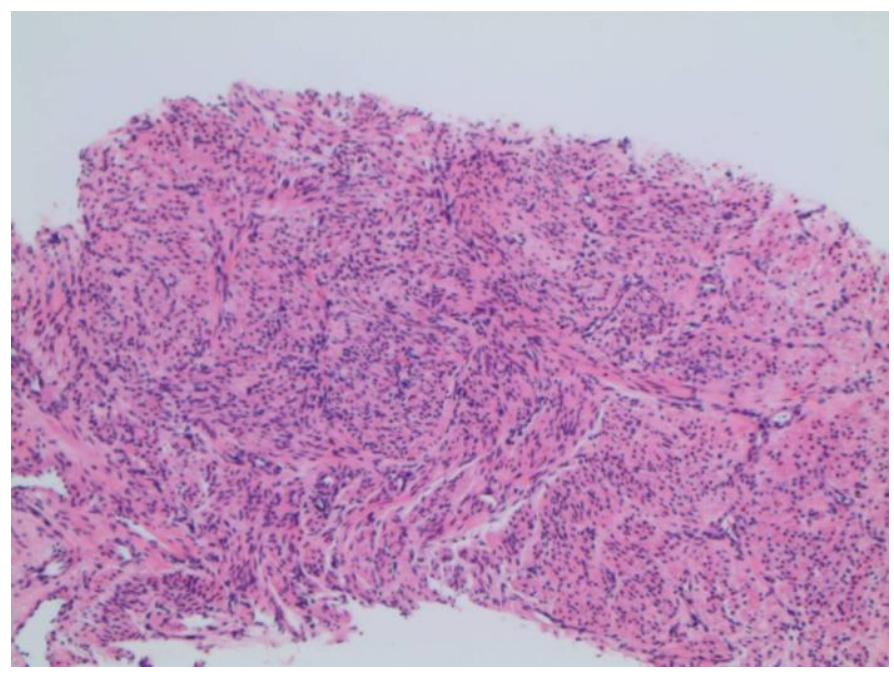

Figure 5: 45 year-old woman with renal leiomyoma.

High power hematoxylin and eosin $(\mathrm{H} \& \mathrm{E})$ slide demonstrating spindle architecture of eosinophilic cells and intersecting fascicles typical of muscle. Several vessels crossing though the sample indicate a vascular tumor.

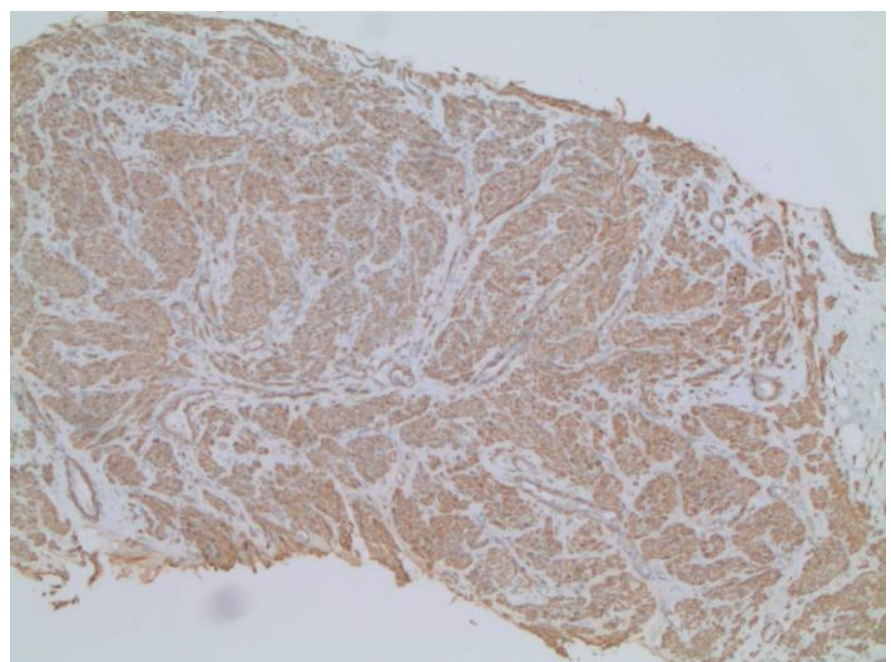

Figure 6: 45 year-old woman with renal leiomyoma.

Diffuse Desmin antibody staining positivity confirms myogenic origin. 


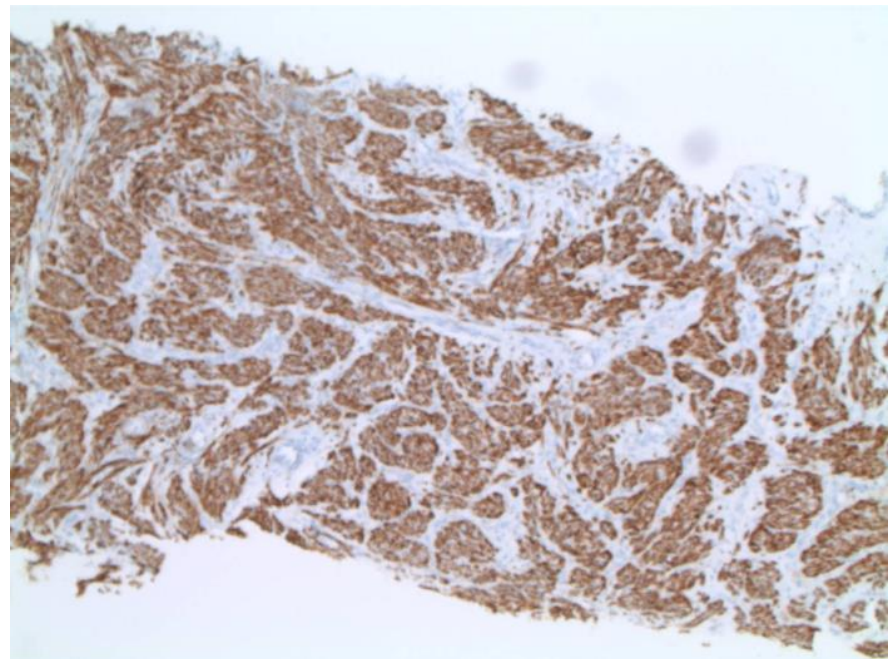

Figure 7: 45 year-old woman with renal leiomyoma.

Diffuse SMA antibody staining positivity confirms smooth muscle origin.

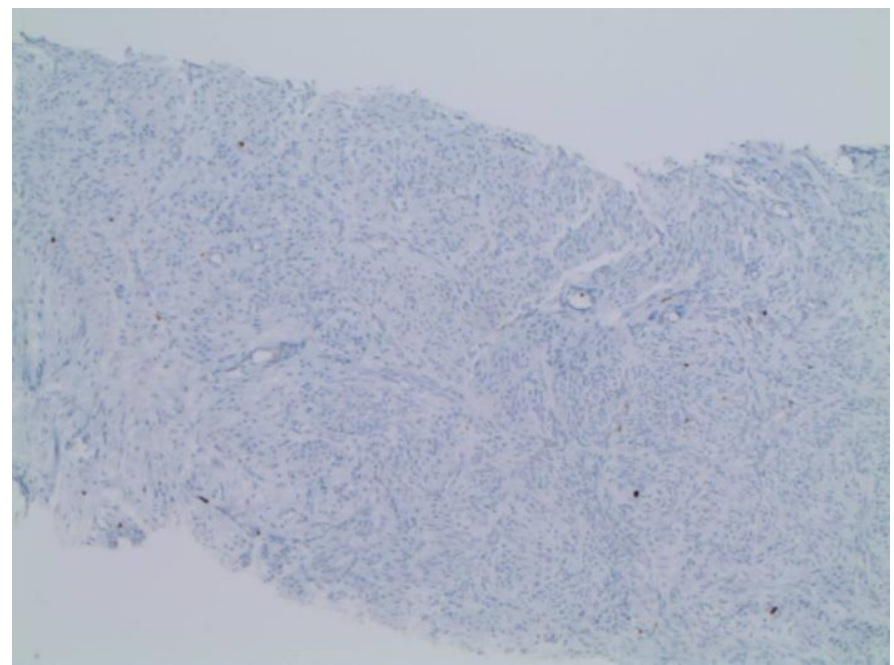

Figure 8: 45 year-old woman with renal leiomyoma.

Sparse Ki-67 antibody staining demonstrates very low cellular proliferation.

\begin{tabular}{|l|l|}
\hline Etiology & Benign proliferation of well differentiated smooth muscle cells \\
\hline Incidence & Constitute only 0.29\% of all treated renal tumors however commonly found on autopsy ( 5\%) \\
\hline Gender ratio & Female predilection of $2: 1$ \\
\hline Age predilection & $\begin{array}{l}\text { Mean age of presentation is } 42 \text { years } \\
\text { Typically presents in females of reproductive age. }\end{array}$ \\
\hline Risk factors & Female gender - possibly due to estrogen and progesterone receptor positivity. \\
\hline Treatment & Surgical resection. If asymptomatic, observation may be appropriate. \\
\hline Prognosis & Excellent \\
\hline Imaging findings & $\begin{array}{l}\bullet \text { US - Hypoechoic mass with well-defined and regular margins } \\
\text { CT non contrast - hyperdense compared to renal parenchyma with density comparable to muscle and } \\
\text { homogeneous }\end{array}$ \\
& $\begin{array}{l}\text { CT IV contrast - hypoenhancing relative to renal parenchyma with progressive enhancement in later } \\
\text { stages and homogeneous } \\
\text { - MRI - Homogeneous low signal intensity on T1 and T2 weighted images }\end{array}$ \\
\hline
\end{tabular}

Table 1: Summary table of renal leiomyoma. 


\begin{tabular}{|c|c|c|c|c|c|}
\hline & Incidence & Clinical presentation & $\begin{array}{l}\text { Imaging features } \\
\text { (US) }\end{array}$ & $\begin{array}{l}\text { Imaging features } \\
\text { (CT) }\end{array}$ & $\begin{array}{l}\text { Imaging features } \\
\text { (MRI) }\end{array}$ \\
\hline Renal leiomyoma & $\begin{array}{l}0.29 \% \text { of all treated } \\
\text { renal tumors } \\
\text { however commonly } \\
\text { found on autopsy } \\
(\sim 5 \%)\end{array}$ & $\begin{array}{l}\text { Pain and a palpable } \\
\text { mass, while less } \\
\text { frequently with } \\
\text { hematuria }\end{array}$ & $\begin{array}{l}\text { Hypoechoic mass } \\
\text { with well-defined } \\
\text { and regular margins }\end{array}$ & $\begin{array}{l}\text { CT - hyperattenuating } \\
\text { relative to renal } \\
\text { parenchyma with } \\
\text { density comparable to } \\
\text { muscle and } \\
\text { homogeneous. } \\
\text { CT contrast - } \\
\text { hypoenhancing } \\
\text { relative to renal } \\
\text { parenchyma }\end{array}$ & $\begin{array}{l}\text { Homogeneous low } \\
\text { signal intensity on } \\
\text { T1 and T2 weighted } \\
\text { images }\end{array}$ \\
\hline $\begin{array}{l}\text { Fat poor-renal } \\
\text { angiomyolipoma } \\
\text { hyperattenuating } \\
\text { subtype }\end{array}$ & $\begin{array}{l}\text { 4-5\% of all AMLs. } \\
\text { Clinically } \\
\text { insignificant AMLs } \\
\text { are common }(0.44 \% \\
\text { prevalence in general } \\
\text { population) }\end{array}$ & $\begin{array}{l}\text { Flank pain, hematuria, } \\
\text { spontaneous } \\
\text { retroperitoneal } \\
\text { hemorrhage, anemia, } \\
\text { urinary tract infection } \\
\text { and renal failure }\end{array}$ & Isoechoic & $\begin{array}{l}\text { CT -hyperattenuating } \\
\text { relative to the renal } \\
\text { parenchyma }\end{array}$ & $\begin{array}{l}\mathrm{T} 1 \text { and } \mathrm{T} 2 \\
\text { hypointense }\end{array}$ \\
\hline Leiomyosarcoma & $\begin{array}{l}\text { Less than } 3 \% \text { of all } \\
\text { malignant renal } \\
\text { tumors }\end{array}$ & $\begin{array}{l}\text { Abdominal/lumbar } \\
\text { pain, palpable mass, } \\
\text { vomiting, hematuria, } \\
\text { and weight loss }\end{array}$ & $\begin{array}{l}\text { Heterogeneous } \\
\text { echotexture }\end{array}$ & $\begin{array}{l}\text { Enhance } \\
\text { heterogeneously } \\
\text { commonly with } \\
\text { evidence of } \\
\text { hemorrhage, necrosis, } \\
\text { and cystic change. }\end{array}$ & $\begin{array}{l}\text { Heterogeneous } \\
\text { signal intensity with } \\
\text { areas of low signal } \\
\text { intensity on T1- and } \\
\text { T2-weighted images }\end{array}$ \\
\hline
\end{tabular}

Table 2: Differential diagnosis table for renal leiomyoma.

\section{ABBREVIATIONS}

ACR $=$ American College of Radiology

$\mathrm{AML}=$ Angiomyolipoma

$\mathrm{CT}=$ Computed Tomography

$\mathrm{H} \& \mathrm{E}=$ Hematoxylin \& Eosin

IV = Intravenous

MRI = Magnetic Resonance Imaging

SMA $=$ Smooth Muscle Actin

TSC $=$ Tuberous Sclerosis Complex

\section{KEYWORDS}

renal leiomyoma; angiomyolipoma; abdominal mass; diagnosis; retroperitoneal; genitourinary; kidney; ultrasound; computed tomography; angiography; mass effect

\section{Online access}

This publication is online available at:

www.radiologycases.com/index.php/radiologycases/article/view/4125

\section{Peer discussion}

Discuss this manuscript in our protected discussion forum at: www.radiolopolis.com/forums/JRCR

\section{Interactivity}

This publication is available as an interactive article with scroll, window/level, magnify and more features.

Available online at www.RadiologyCases.com

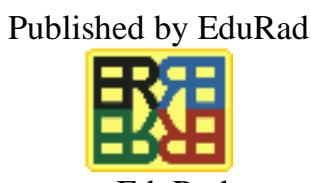

www.EduRad.org 